\title{
Technology and Developments for the Random Positioning Machine, RPM
}

\author{
A. G. Borst • Jack J. W. A. van Loon
}

Received: 11 March 2008 / Accepted: 16 June 2008 / Published online: 3 September 2008

(C) The Author(s) 2008. This article is published with open access at Springerlink.com

\begin{abstract}
A Random Positioning Machine (RPM) is a laboratory instrument to provide continuous random change in orientation relative to the gravity vector of an accommodated (biological) experiment. The use of the RPM can generate effects comparable to the effects of true microgravity when the changes in direction are faster than the object's response time to gravity. Thus, relatively responsive living objects, like plants but also other systems, are excellent candidates to be studied on RPMs. In this paper the working principle, technology and control modes will be explained and an overview of the previously used and available experiment systems will be presented. Current and future developments like a microscope facility or fluid handling systems on the RPM and the option to provide partial gravity control modes simulating for instance Mars or Moon gravity will be discussed.
\end{abstract}

Keywords Random positioning machine . 2D 3D clinostat $\cdot$ Simulated microgravity . Microweight $\cdot$ Hypogravity

\footnotetext{
A. G. Borst ( $\bowtie)$

Dutch Space, Newtonweg 1, 2333 CP Leiden,

The Netherlands

e-mail: a.borst@dutchspace.nl

URL: www.dutchspace.nl

J. J. W. A. van Loon

Dutch Experiment Support Center (DESC),

Dept. Oral Cell Biology, ACTA Vrije Universiteit,

Van de Boechorststraat 7, 1081BT Amsterdam,

The Netherlands

e-mail: j.vanloon@vumc.nl

URL: www.descsite.nl
}

\section{Introduction}

Microgravity simulation by means of continuous change of orientation of objects relative to the gravity's vector can generate effects comparable to the effects of true microgravity when the changes are faster than the objects response time to gravity. Thus, relatively responsive living objects, like plants are excellent candidates to be studied on clinostats, 3D clinostats and the Random Positioning Machine (RPM). The clinostat provides rotation around one horizontal axis. The objects in such a clinostat experience gravity continuously rotating in one vertical plane. Especially for larger objects it was recognized that the 3D random rotation could provide a better simulation of the weightlessness conditions as compared to the classical 2D clinostat (Kraft et al. 2000).

To describe the working principle of an RPM we can use the analog of a sphere fountain, a round marble stone supported by a concave water bearing. We assume the marble perfectly symmetrical around two axis and the water stream providing no rotation forces to the marble. Imagine walking on top of the marble and think of the effect to the geometrical centre of the spherical item. Now the gravity vector points to the opposite direction with respect to the walker.

If walking in a straight line the marble will rotate around one axis (clinostat mode). The walker, and therefore also the imaginary gravity vector, is always crossing one meridian. We can define walk paths over the ball continuously changing the direction of the gravity vector with respect to the sphere's center. But by just varying the orientation we are not sure that this motion is effective in averaging gravity. To effectively simulate microgravity, symmetry in the walk path over 
the sphere is required in order to average the orientation in two axes the walker has to spend the same time on each surface section of the marble.

This concept of a rotating sphere for microgravity simulation was breadboarded in 2001 by a student team at Dutch Space (Fig. 1). Access to the sphere's interior where the sample could be suspended in the centre of the sphere was possible by detaching the two halves. Although the system did work and it was easily possible to create random rotation with the drive system, this project did not lead to a product since it was difficult to produce a segmented sphere with perfectly smooth outer surface. A perfect sphere is required to allow a rotation of the sample without vibrations and linear accelerations. Within this concept it was also difficult to provide power and data connections to the inside of the sphere.

A system that comes close to the rotational freedom of the sphere is the use of two perpendicular and independently driven frames (Fig. 2). Application of this mechanism was first introduced by von Sachs (1879). This concept has been adopted by all the manufacturers of 3D clinostats and RPMs see also the history and the development of the RPM that has been described by van Loon (2007). The discrete rotation axes allow the implementation of slip rings to provide power and exchange data with the experiment that can be mounted onto the inner frame. The control of the two rotating frames is, however, not straightforward. There are a number of control modes that can be selected. We will

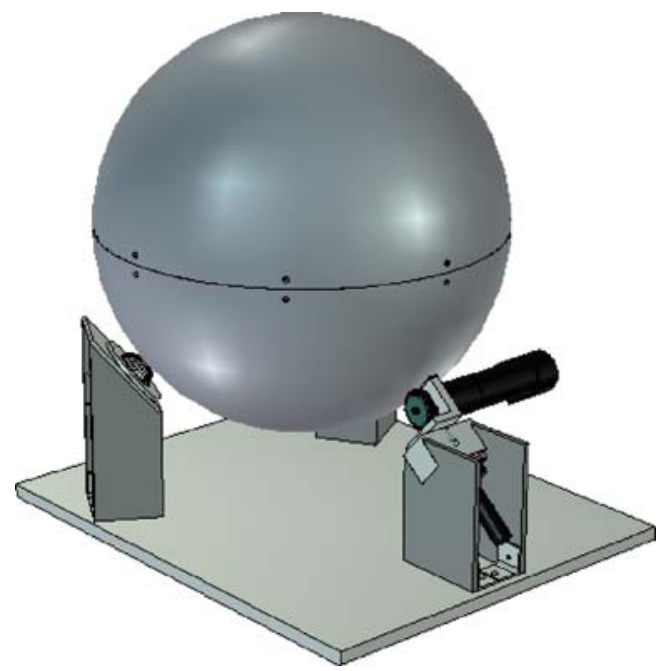

Fig. 1 Ball RPM. The ball RPM is supported on two freely rotating contact points. The third contact point is a wheel connected to a motor that drives the rotation of the large sphere. The orientation of this motor and drive wheel can be varied by a second motor. The combined control of these motors allows a free walk path over the sphere, resulting in a continuously change in orientation of the sphere with its suspended sample system

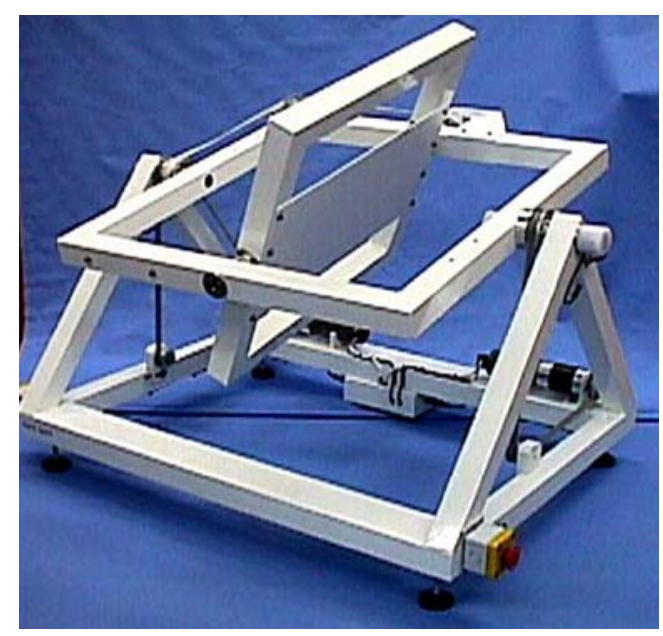

Fig. 2 RPM with two independently driven perpendicular frames

also describe a number of experiment systems which have been developed in order to allow the execution of automated experiments on the RPM.

\section{The RPM Control Modes}

We want to manipulate the sample on the RPM randomly to different orientations, therefore we need to control the RPM in a way no dominant orientation prevails during an experiment run. This can be achieved in two ways. The walk pattern can be made regular with, as input parameter, the even distribution of orientations. Another approach is to use the random walk pattern. When the walk path is truly random, the path coverage over the sphere surface will become equally distributed after a long time. The use of the random walk control principle has also the advantage that the rotation does not expose a predictable motion environment to the specimen which could be relevant since some living organisms might adapt to such a predictable motion pattern.

\section{Clinostat Mode}

The first mode is the classical clinostat mode. In this setting the RPM control drives only one of the two frames with a constant speed. The other axis is oriented horizontally (Fig. 3a, b).

\section{D Clinostat Mode}

In the 3D clinostat mode (Fig. 4a, b) the two axes are rotated at a constant speed. Although this is a $3 \mathrm{D}$ motion, it is clear that the $3 \mathrm{D}$ clinostat based on two perpendicular frames does not provide a symmetric 


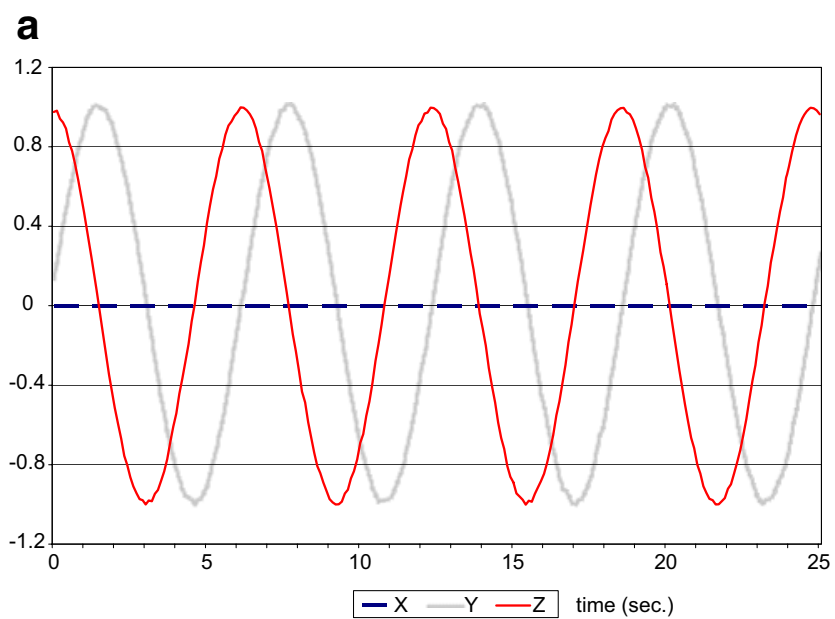

b

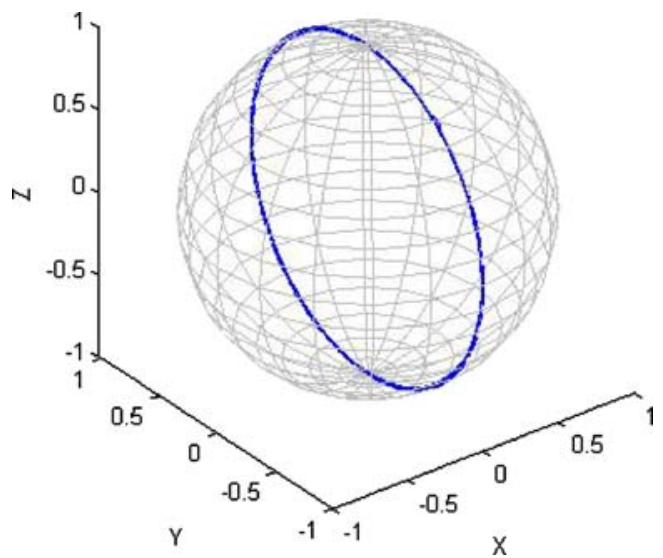

Fig. 3 a Gravity values in a standard clinostat mode. $Y$ and $Z$ are changing sinusoidal between $+1 \mathrm{~g}$ and $-1 \mathrm{~g}$. b The clinostat mode results in a $2 \mathrm{D}$ motion trajectory, in this graph projected on an imaginary sphere. The abscissa in a as well as the three axes in b are accelerations expressed as ' $\mathrm{g}$ '. Time displayed in seconds is displayed at the ordinate in $\mathbf{b}$

coverage of all orientations. Such a trajectory is repetitive and predictable, therefore random motion is to be introduced in order to cover all possible orientations.

\section{D Random Mode}

With random mode settings the individual frame speed and in this example the rotation direction are randomly varied this results an unpredictable and symmetrical path. See Fig. 5a, b. In 1986 Japanese researchers developed the first modern version of a $3 \mathrm{D}$ clinostat used initially for plant research (Murakami and Yamada 1988; Hoson et al. 1992, 1997).

In 1994 the notion of "true random positioning" was advocated by Dr. D Mesland of the European Space Agency, ESA (Mesland 1996). Dutch Space developed the first prototype instrument using the random walk a

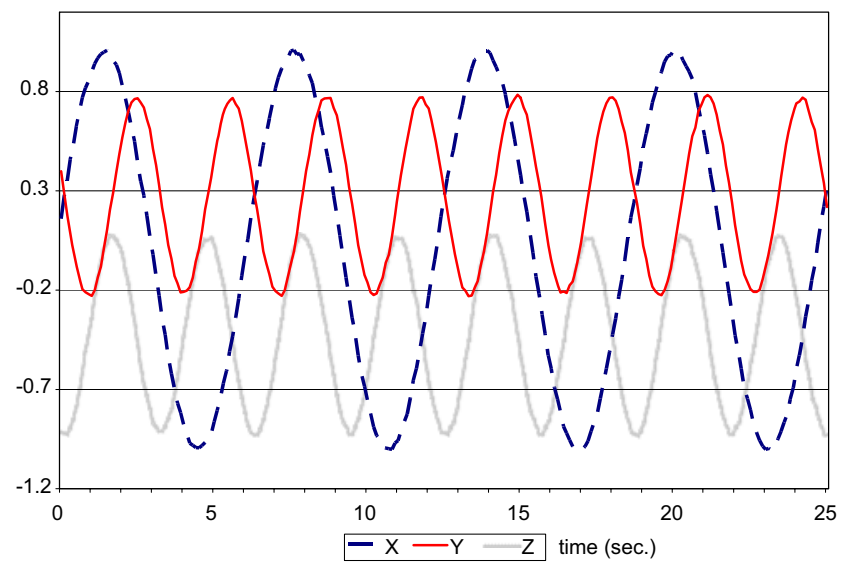

b

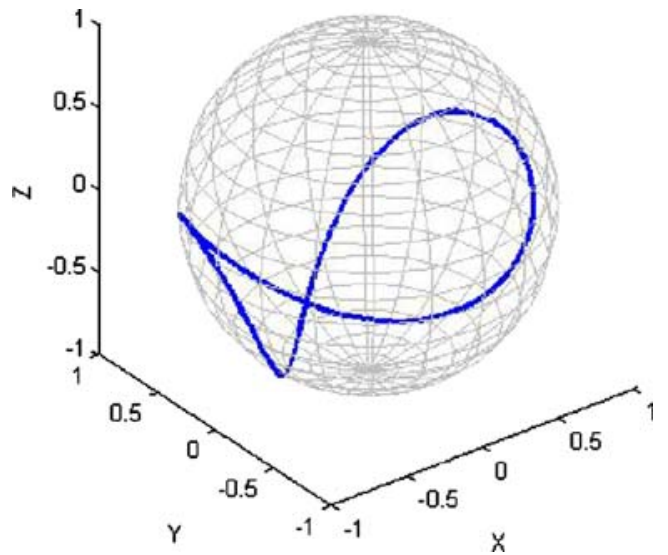

Fig. 4 a Gravity values measured on the RPM, rotated in 3D clinostat mode, the inner and outer frame rotate (in this example) both at a constant speed of $60^{\circ} / \mathrm{s}$. b Motion trajectory 3D clinostat mode with constant speed for both frames of $60^{\circ} / \mathrm{s}$, displayed on an imaginary sphere. The abscissa in $\mathbf{a}$ as well as the three axes in b are accelerations expressed as ' $\mathrm{g}$ '. Time displayed in seconds is displayed at the ordinate in $\mathbf{b}$

principle. The current control of the RPM provides different operational modes to apply such parameters (see earlier).

It is important to accommodate an experiment exactly in the center of an RPM. The rotation of the sample induces also centripetal acceleration. Placing the sample in the centre of the RPM this effect will be negligible but when using larger samples volumes or multiple samples especially in combination with high rotation speeds these residual accelerations may become relevant (van Loon 2007).

Finally an option is provided to generate a file with control parameters that can be reviewed and re-used; a pre-generated random scenario. This random file option allows repeating experiments with the same random walk sequence each time. 


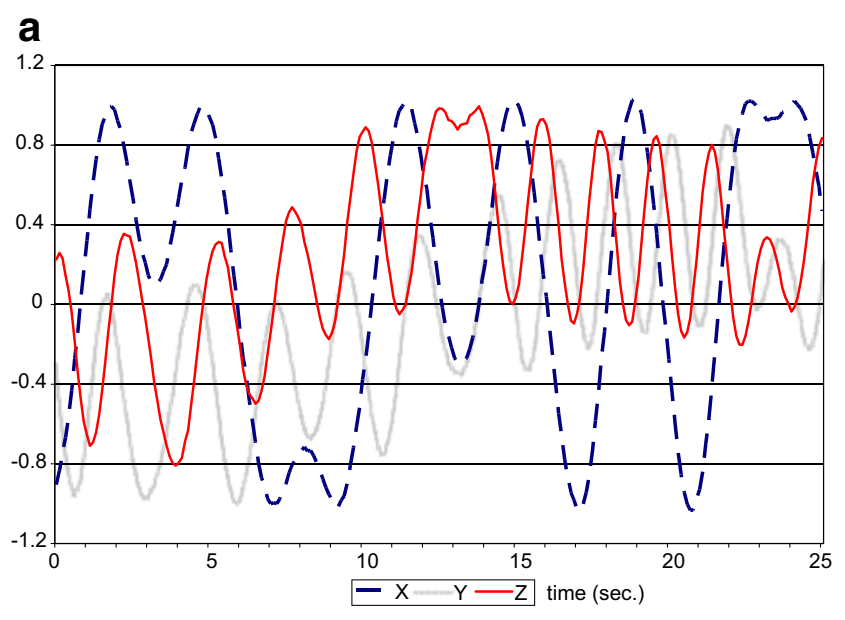

b

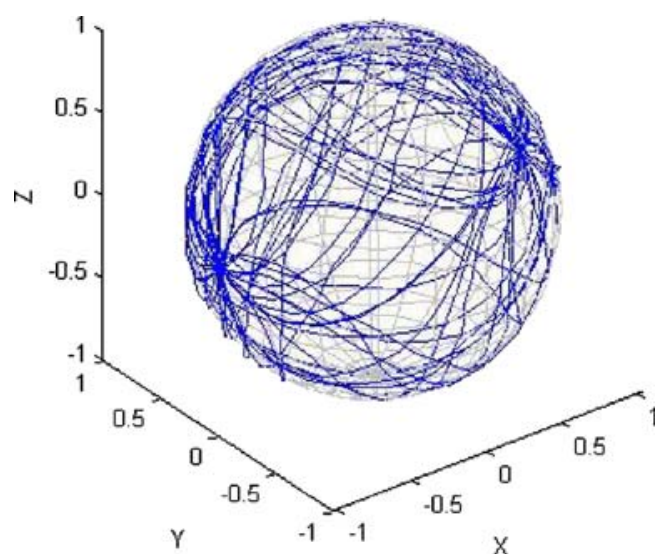

Fig. 5 a Gravity values measured on the RPM, rotated in random speed and random direction mode, the inner and outer frame rotate (in this example) are at a varying speed between $60-120^{\circ} / \mathrm{s}$. It should be noted that most users apply a speed of $+/-60^{\circ} / \mathrm{s}$ for both frames. b Motion trajectory in random speed and random direction mode, displayed on an imaginary sphere. The abscissa in $\mathbf{a}$ as well as the three axes in $\mathbf{b}$ are accelerations expressed as ' $\mathrm{g}$ '. Time displayed in seconds is displayed at the ordinate in $\mathbf{b}$

\section{Experiment Accommodation}

Experiment Control during RPM Experiments

The performance of an experiment on the rotating RPM often requires interaction with the samples on the experiment platform in order to e.g. activate and fix samples. With the COBRA (COmpact BioReactor Assembly) system, actuators on the platform can be operated while the RPM is running. A test set up consisting of actuators (commercial valves, pumps, motors, heaters etc.) including their feedback can be operated via a Windows based personal computer. Communication and power is provided via the sliprings of the RPM.
Running a simple script allows the execution of an accurate and repeatable timeline. The same equipment but not installed on the RPM can be used as 1-g reference.

When using liquids on the RPM we have to fill the experiment volume without gas bubbles and avoid loss of liquid during rotation. Gas bubbles result in unwanted fluid motion and associated shear stress to the sample due to the moving gas bubbles in the liquid volume (van Loon 2007). Fluid motion will not only be driven by density differences of the rotated volume, but also by the inertia of the fluid in combination with the accelerations of the RPM. It is therefore proposed to choose a motion profile with low accelerations. As 1-g control experiment it would be useful to expose the sample to comparable fluid motion, as can be simulated performing a motion profile around the vertical axis with the same accelerations as has been used in the $3 \mathrm{D}$ random mode.

The COBRA is based on the use of commercial available microslides, multiwells, syringes, and two peristaltic pumps provide the fluid exchanges (Fig. 6). A combined septa/seal provides leak-tightness for the multiwells and allows gas free filling of the experiment volume.

\section{Temperature Control}

For most experiments that are performed on the RPM, temperature control and often also humidity control are important. To control these parameters there are two options. The first is the installation of the complete RPM into an incubator as can be seen in Fig. 7. This is the most used and most straightforward solution. Therefore the desktop RPM has been designed to fit

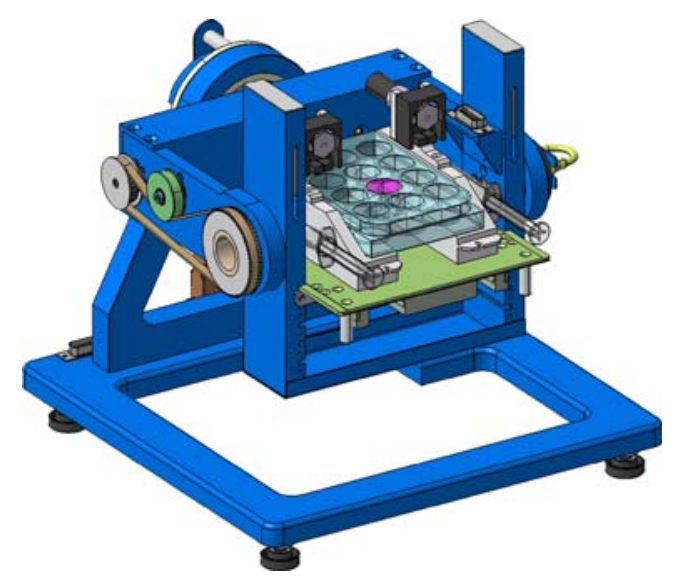

Fig. 6 COBRA facility on a desktop RPM. In this set up fibroblasts cells were used attached to round micro slides placed in the central cell of the multiwell plate 


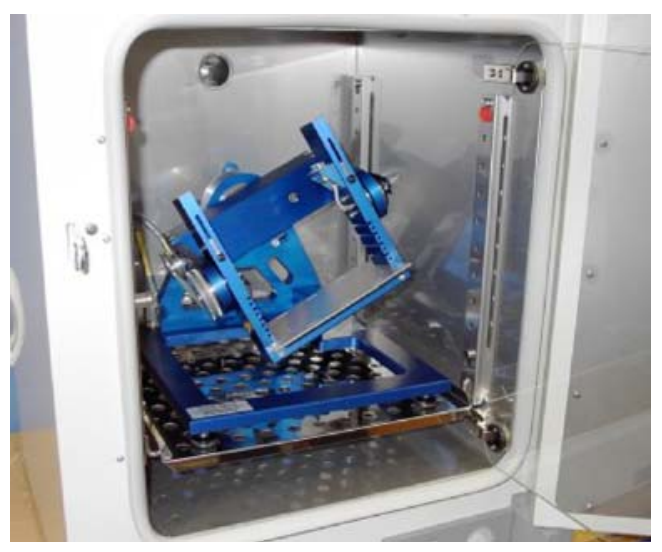

Fig. 7 Desktop RPM in a standard incubator

into a $50 \times 50 \times 50 \mathrm{~cm}$ incubator. To allow easy integration in a commercial incubator we can make use of the standard available electrical feed through in the back wall in order to connect the RPM to its controller. The RPM functions well when exposed to temperatures up to $40^{\circ} \mathrm{C}$ even in a high humidity (non condensing) environment.

The other option for experiment temperature control is the installation of a small incubator on the RPM platform. This option can be used for temperature environments that are not compatible with RPM requirements $\left(>40^{\circ} \mathrm{C}\right.$ and a condensing humidity environment). One can also make use the small thermal inertia of the local incubator to control a dedicated temperature profile. Long duration microgravity experiments like in the International Space Station (ISS) often provide a temperature environment that can be controlled during the actual experiment but not during the whole mission. After the mission the temperature history can be reproduced. The flight experiment can be repeated in order to have a well matched 1-g ground control or an RPM (simulated flight) experiment. The TAIPAN (Temperature Accommodation Incubator for Post flight Analysis) provides the means to control the temperature for RPM experiments. The configuration in Fig. 8 shows a TAIPAN for a set of four standard ESA Type I/E experiment containers used in parallel. The facility also provides power interfaces to the experiment units simulating for instance a Kubik or Biobox facility. A first evaluation model of the TAIPAN was produced for the Biokin experiment from Dr. Krooneman (BioClear, Groningen, The Netherlands) launched in October 2007. This experiment is sensitive to temperature variations also during transport. The ground reference experiments (both $1 \mathrm{~g}$ and simulated micro-g) after the actual flight will be performed with actual flight transport and in-flight temperature profile.

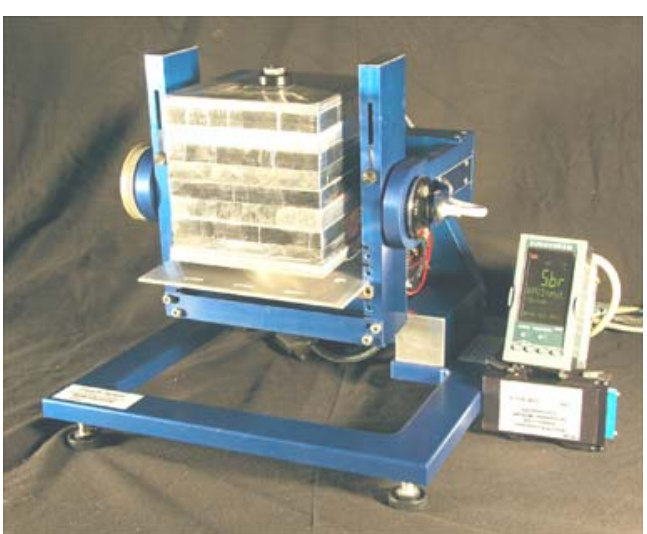

Fig. 8 The temperature controllable TAIPAN facility on the desktop RPM

\section{Technological Developments}

There are two strong demands from the users of RPMs. One is to allow microscopy on the RPM and the other is the wish to allow experiments at partial g-levels (between 0 and $1 \mathrm{~g}$ ).

\section{RPM Microscopy}

As is the case for most flight experiments the analysis of any microgravity effects is done after fixation of the specimens. It would be very relevant to monitor the experiment during actual microgravity. Especially

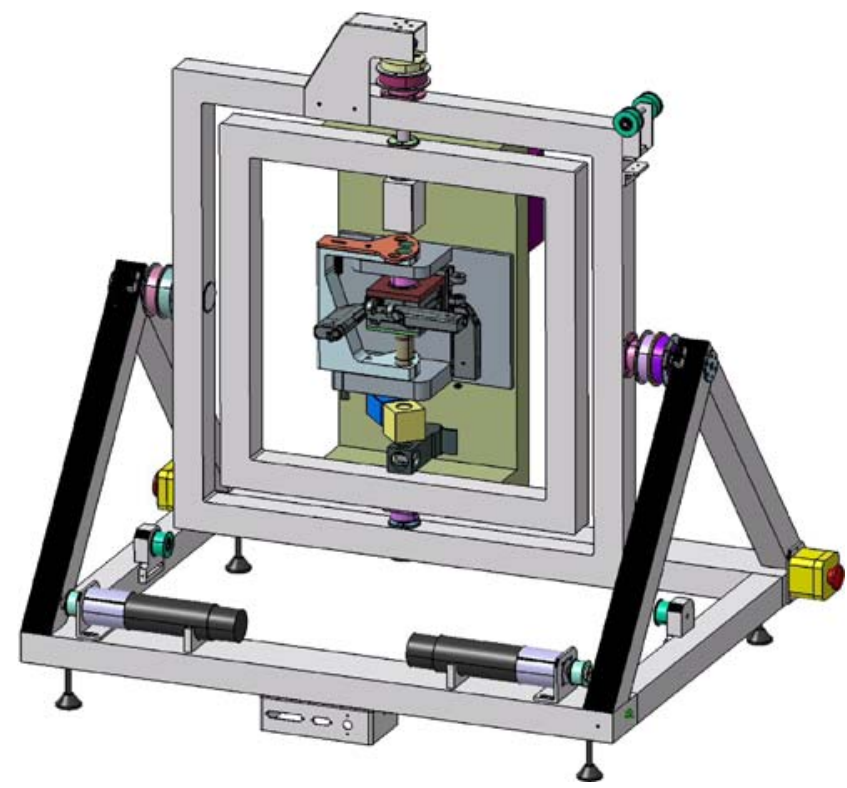

Fig. 9 A newly designed fluorescent/confocal microscope mounted onto a full size RPM 
with the developments like GFPs (Green Fluorescent Protein) it is possible to monitor living cells and tissues in vitro. This opens possibilities to look at possible microgravity effects in real time during the course of the incubation time instead of only having the end point samples after fixation. Regarding ISS experiments this would require a microscope facility in the ISS. For simulated microgravity a microscope on the RPM would be an option. Currently we are investigating the possibilities of confocal microscopy on a full size RPM (Fig. 9).

\section{Future Developments}

On Earth 1-g as well as hyper gravity experiments can relatively easily be performed. Simulated micro-g experiments are more difficult but can be done as described above. Experiments between 0 and 1 -g require, for instance, a centrifuge running in a microgravity environment. Such experiments have been performed in centrifuges in e.g. the Biorack (Mesland et al. 1987; Brillouet et al. 1995), the NIZEMI (Friedrich et al. 1996) and the Biopack (van Loon 2004).

It would be interesting to have the possibility to generate simulated hypogravity to mimic for instance 0.38-g (Mars) or 0.17-g (Moon) gravity levels. This allows studying biological processes in support of future planetary exploration programs. Another more fundamental research application is to use the partial $\mathrm{g}$ function to determine the threshold for gravity response of various organisms.

\section{Conclusion}

Since the introduction of the very first machine to nullify gravity by von Sachs in 1879 various 2D and 3D clinostats and RPM's have been developed (van Loon 2007). The wealth of scientific publications using these systems demonstrate the valuable contribution of such technology in our understanding of the effects of gravity on living systems. The systems are used for stand alone studies or in preparation for real space flight experiments (van Loon et al. 1999). Based on this further developments are needed to increase the experimental setup possibilities of such devices by implementing systems for automated fluid handling or advanced microscopy.
Acknowledgements This paper was possible with support from NWO-ALW via the Netherlands Institute for Space Research, SRON Grant MG-057 (van Loon).

Open Access This article is distributed under the terms of the Creative Commons Attribution Noncommercial License which permits any noncommercial use, distribution, and reproduction in any medium, provided the original author(s) and source are credited.

\section{References}

Brillouet, C., Brinckmann, E., Stavros, E.L.: An overview of the operation and results of the Biorack experiments on the IML-1 mission. In: Mattok, C. (ed.) Biorack on Spacelab IML-1. SP-1162. ESA Publication Div. ESTEC, Noordwijk (1995)

Friedrich, U.L., Joop, O., Pütz, C., Willich, G.: The slow rotating centrifuge microscope NIZEMI-a versatile instrument for terrestrial hypergravity and space microgravity research in biology and materials science. J. Biotechnol. 47(2-3), 225-38 (1996), 27 June

Hoson, T., Kamisaka, S., Masuda, Y., Yamashita, M.: Changes in plant growth processes under microgravity conditions simulated by a three-dimentional clinostat. Bot. Mag. 105, 53-70 (1992)

Hoson, T., Kamisaka, S., Masuda, Y., Yamashita, M., Buchen, B.: Evaluation of threedimentional clinostat as a simulator of weightlessness. Planta 203, 187-197 (1997)

Kraft, T.F.B., van Loon, J.J.W.A., Kiss, J.Z.: Plastid position in Arabidopsis columella cells is similar in microgravity and on a random-positioning machine. Planta 211(3), 415-422 (2000)

Mesland, D.A.: Novel ground-based facilities for research in the effects of weight. ESA Microgravity News 9, 5-10 (1996)

Mesland, D., Brillouet, C., et al.: SP-1091. In: Longdon, N., David, V. (eds.) Biorack on Spacelab D-1. ESA Publication Div. ESTEC, Noorwijk (1987)

Murakami, S., Yamada, M.: Architecture of statocytes and chloroplasts under the microgravity environment. Jap. Soc. Biol. Sci. Space 2(4), 301 (1988)

van Loon, J.J.W.A.: The ground controlled late access biological research facility. J. Grav. Phys. 11(1), 57-65 (2004)

van Loon, J.J.W.A.: Some history and the use of Random Positioning Machine, RPM, in gravity related research. Adv. Space Res. 39, 1161-1165 (2007)

van Loon, J.J.W.A., Veldhuijzen, J.P., Kiss, J., Wood, C., vd Ende, H., Guntemann, A., Jones, D., de Jong, H., Wubbels, R.: Microgravity research starts on the ground! Apparatusses for long term ground based hypo- and hypergravity studies. Proc. 2nd European Symp. on the Utilisation of the International Space Station, ESTEC, pp. 415-419. Noordwijk, The Netherlands, 16-18 November 1998. ESA SP-433 (1999)

von Sachs, F.G.J.R.: Ueber Ausschliessung der geotropischen und heliotroposchen Krümmungen wärend des Wachsthums. Würzburger Arbeiten 2, 209-225 (1879) 